\title{
Saccade Planning Evokes Topographically Specific Activity in the Dorsal and Ventral Streams
}

\author{
Golbarg T. Saber, ${ }^{1 *}$ Franco Pestilli, ${ }^{3 *}$ and Clayton E. Curtis ${ }^{1,2}$ \\ ${ }^{1}$ Center for Neural Science, and ${ }^{2}$ Department of Psychology, New York University, New York, New York 10003, and ${ }^{3}$ Department of Psychological and Brain \\ Sciences, Indiana University, Bloomington, Indiana 47405
}

\begin{abstract}
Saccade planning may invoke spatially-specific feedback signals that bias early visual activity in favor of top-down goals. We tested this hypothesis by measuring cortical activity at the early stages of the dorsal and ventral visual processing streams. Human subjects maintained saccade plans to (prosaccade) or away (antisaccade) from a spatial location over long memory-delays. Results show that cortical activity persists in early visual cortex at the retinotopic location of upcoming saccade goals. Topographically specific activity persists as early as V1, and activity increases along both dorsal (V3A/B, IPS0) and ventral (hV4, V01) visual areas. Importantly, activity persists when saccade goals are available only via working memory and when visual targets and saccade goals are spatially disassociated. We conclude that top-down signals elicit retinotopically specific activity in visual cortex both in the dorsal and ventral streams. Such activity may underlie mechanisms that prioritize locations of task-relevant objects.
\end{abstract}

Key words: antisaccade; fMRI; retinotopy; saccade; spatial cognition; working memory

\section{Introduction}

Neural activity persists in frontal cortex during the maintenance of items in working memory (Funahashi et al., 1989; Srimal and Curtis, 2008), when attention is deployed covertly (Kastner et al., 1999), and while a saccade is planned to acquire a target (Curtis and Connolly, 2008). Theoretically, persistent activity in frontal cortex may reflect top-down feedback signals that bias the competition for neural representation in visual cortex (Desimone and Duncan, 1995; Corbetta et al., 2002). For example, a location can be prioritized by the pattern of activity within a population of spatially tuned neurons in prefrontal cortex. The read-out of this activity, can be thought of as an attention pointer, and may provide the means by which visual neurons with receptive fields matching the locus of the objects of priority are favored. Indeed, stimulation of the frontal eye field (FEF) in the frontal lobe causes both behavioral and neural effects in visual cortex consistent with this idea (Moore and Armstrong, 2003; Moore and Fallah, 2004). This has led to the belief that the mechanisms by which attention is controlled has evolved within or co-opted oculomotor systems. Shifting one's attention shares the neural machinery for shifting

\footnotetext{
Received April 26, 2014; revised Nov. 6, 2014; accepted Nov. 10, 2014

Author contributions: G.T.S. and C.E.C. designed research; G.T.S. and C.E.C. performed research; G.T.S., F.P., and C.E.C. analyzed data; G.T.S., F.P., and C.E.C. wrote the paper.

This work was supported by R01-EY016407 to C.E.C. We thank the NYU Center for Brain Imaging's Pablo Velasco, Keith Sanzenbach, and Valerio Luccio for help with data collection; Shani Offen and David Heeger for helpful discussions on data analyses; Justin Gardner for help with analytic software; Jonathan Winawer for help with ventral visual mapping; Wayne Mackey for help scoring the saccade behavior; and Marisa Carrasco and Sam Ling for comments on early versions of the paper.

The authors declare no competing financial interests.

${ }^{*}$ G.T.S. and F.P. contributed equally to this work.

Correspondence should be addressed to Clayton Curtis, New York University, 6 Washington Place, Room 863, New York, NY 10003. E-mail: clayton.curtis@nyu.edu.

DOI:10.1523/JNEUROSCI.1687-14.2015

Copyright $\odot 2015$ the authors $\quad 0270-6474 / 15 / 350245-08 \$ 15.00 / 0$
}

one's gaze (Awh et al., 2006). Remembering a location shares the neural machinery that controls the locus of attention (Awh and Jonides, 2001). Indeed, activity in monkey V1 neurons with receptive fields matching the goal of a memory-guided saccade persists during saccade preparation (Supèr et al., 2001). Here we test whether this critical finding also occurs in humans. To date, neuroimaging studies have almost unanimously reported that activity in human early visual cortex does not persist above pretrial baseline levels when maintaining feature information across working memory delays (Ester et al., 2009; Offen et al., 2009; Serences et al., 2009; Riggall and Postle, 2012; Albers et al., 2013).

Here, we used functional magnetic resonance imaging (fMRI) to measure cortical activity in human retinotopic visual areas $\mathrm{V} 1$, V2, V3, hV4, VO1, V3A/B, and IPS0 while subjects maintained over long memory delays planned saccades to visual targets. Furthermore, we tested for the existence of topographically specific saccade planning activity in the absence of visual stimulation by disassociating the retinotopic locus of top-down and bottom-up stimulation using a memory-guided antisaccade task.

We demonstrate that cortical activity persists during saccade preparation periods at the retinotopic location of memorized saccade goals. Activity persisted at the saccade-goal representation in early visual cortex $(\mathrm{V} 1-\mathrm{V} 3)$ and in the dorsal stream (V3A/B and IPS0). V3A/B and IPS0 are areas involved in spatial orienting and saccade preparation (Schluppeck et al., 2005; Silver et al., 2005; Kastner et al., 2007; Swisher et al., 2007; McKeefry et al., 2008; Fischer et al., 2012). Importantly, activity also persisted at the retinotopic location of planned saccades in ventral stream visual areas (hV4 and VO1) traditionally believed to be involved primarily in object, feature, and form processing (Bartels and Zeki, 2000; Brewer et al., 2005; Arcaro et al., 2009). 
A
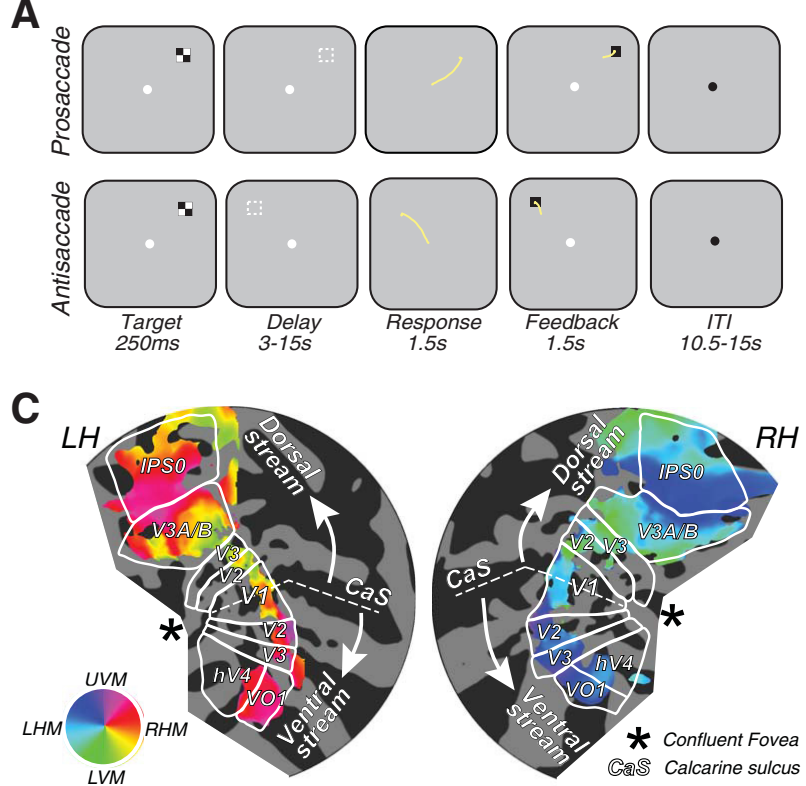

B
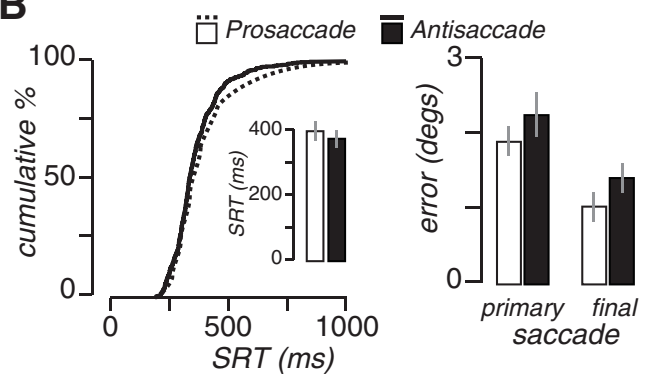

D

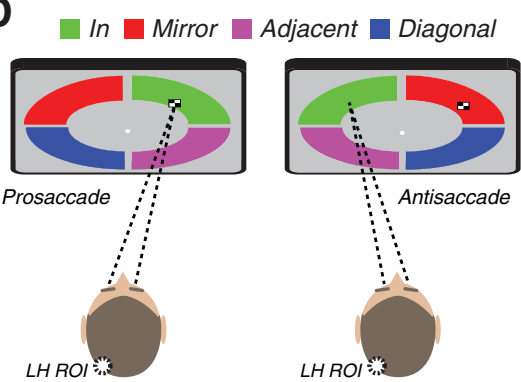

Figure 1. Experimental task, behavioral data, and topographic mapping. A, Trial schematics of memory-guided prosaccade and antisaccade tasks. The dashed square depicted during the delay epochs represent the saccade goal, but were not visible during the task. $\boldsymbol{B}$, Neither SRTs nor saccade errors differed significantly between the tasks. Error bars show SEM across subjects. C, Definition of retinotopic visual areas projected onto flattened occipital-parietal patches from an example subject. The colors depict the phase estimates from the localizer task that mapped the polar angle within a ring $9-11^{\circ}$ in the periphery where saccades were planned in the saccade tasks. LH, Left hemisphere; RH, right hemisphere; UVM, upper visual meridian; LVM, lower visual meridian; LHM, left horizontal meridian. $\boldsymbol{D}$, Delay period data extracted from retinotopic ROls mapping quadrant bands were sorted into four trial types according to the quadrant into which the saccade was planned. We compared trials in which the saccade plan was directed into the part of the visual field mapped by the topographic ROI (In, green) with trials in which it was directed into other visual field quadrants [i.e., the mirror image location in the opposite hemifield (red), the adjacent quadrant in the same hemifield (magenta), and the diagonal quadrant (blue)]. The example shown depicts a left hemisphere ROI that maps the contralateral upper visual field quadrant.

\section{Materials and Methods}

Subjects. Five healthy subjects ( 3 female; right-handed; $25-42$ years old) participated after giving informed consent, using procedures approved by New York University. Each subject participated in six scanning sessions: one to obtain three high-resolution anatomies, one to measure retinotopy, and four to measure responses during memory-guided prosaccade and antisaccade tasks.

Memory-guided saccade tasks. Subjects performed two memoryguided saccade tasks (Fig. 1A). Subjects were instructed to remember the location of a briefly presented visual target and immediately plan a saccade to, in the case of memory-guided prosaccades, or away from, in the case of memory-guided antisaccades, a high-contrast flickering visual target ( $1^{\circ}$ diameter, spatial frequency $2 \mathrm{cpd}, 8 \mathrm{~Hz}$ flicker) that appeared at one of eight locations separated by $30^{\circ}$ angles at $10^{\circ}$ eccentricity, avoiding vertical and horizontal meridians. Each trial began with $1.25 \mathrm{~s}$ of central fixation followed by a brief presentation of a visual target. Subjects maintained the planned prosaccade or antisaccade over a long and variable delay period (3-15 s). Disappearance of the fixation along with a brief auditory cue prompted the memory-guided saccade as accurately and quickly as possible. Subjects held their gaze until the correct location was presented as feedback and corrected any error in their gaze. Subjects then returned to fixation for the intertrial interval (10.5-15 s). Partial trials were also included to help break the dependency of the delay on the target. These trials were aborted after the visual target, signaled by a change in the color of the fixation spot. In four sessions, each with eight 322 s scans, subjects performed 192 prosaccades and 192 antisaccades trials, blocked by condition.

Oculomotor methods. Eye position was recorded in the scanner at 1000 $\mathrm{Hz}$ (EyeLink 1000, SR Research). We computed both error in degrees of angle of the primary saccade and the final error after any quick corrections were made before the target feedback. All trials were inspected for noncompliance. Trials in which subjects made an eye movement to the wrong saccade target were rare $(0.3 \%$ and $0.6 \%$ of all prosaccade and antisaccade trials, respectively). Trials where gaze deviated from fixation $>2^{\circ}$ during the delay-period or immediately following visual target
( $4.6 \%$ and $2.7 \%$ of prosaccade and antisaccade trials, respectively) were excluded from analysis.

MRI acquisition. All MRI data were acquired on a 3T Allegra head-only scanner (Siemens) using a head transmit coil (NM-011) and two surface receive coils (NOVA Medical): (1) a four-channel phased array receive surface coil (NMSC-021) for retinotopic mapping, and (2) a fourelement phased parallel array (NMSC-011) for memory-guided saccade tasks. We acquired $\mathrm{T}^{*}$-sensitive echoplanar images [repetition time (TR) $1.5 \mathrm{~s}$; echo time $30 \mathrm{~ms}$; flip angle $75^{\circ}$; 26 slices; $3 \times 3 \times 3 \mathrm{~mm}$ voxels; $192 \times 192 \mathrm{~mm}$ field-of-view]. Three T1-weighted MPRAGE scans were averaged and were used for gray matter segmentation, cortical flattening, registration, and visualization for creating regions-of-interest (ROIs; see next two sections). Functional scans were corrected for head motion and aligned across sessions, detrended, and high-pass filtered with a cutoff frequency of $0.0167 \mathrm{~Hz}$, and converted to percentage signal modulation.

Retinotopic mapping. Retinotopic visual areas (V1, V2, V3, hV4, VO1, $\mathrm{V} 3 \mathrm{~A} / \mathrm{B}$, IPS0) were defined with standard phase encoded approach (Wandell et al., 2007). Polar angle components of the retinotopic maps were estimated with a high contrast flickering wedge that rotated (clockwise or counterclockwise) around central fixation (subtended $90^{\circ}$ of polar angle). Radial components were estimated with a high contrast flickering annulus expanding or contracting from fixation (subtended $4.2^{\circ}$ radius). Each scan consisted of 10.5 cycles with a period of $24 \mathrm{~s}$, and lasted $252 \mathrm{~s}$. Each subject completed six to eight runs of the rotating wedge aperture and four runs of expanding/contracting annulus aperture.

Retinotopic saccade localizer. We then identified the precise portions of retinotopic maps that matched the location of the saccade targets from the memory-guided saccade tasks using a localizer. We modified the rotating wedge stimulus (flickering checkerboard originally used for retinotopic mapping) so that its diameter was set to $2^{\circ}$ and it extended from $9^{\circ}$ to $11^{\circ}$ eccentricity. Recall the eccentricity of saccade targets was $10^{\circ}$. The size of rectangular elements in the checkerboard was decreased from $5^{\circ}$ to $1^{\circ}$, and their radius size was decreased from $2^{\circ}$ to $1^{\circ}$. Each scan consisted of 10.5 cycles of length $24 \mathrm{~s}$ and lasted $252 \mathrm{~s}$. Overall, 8-14 
localizer scans were collected across four scanning sessions for each subject.

Defining retinotopic areas. We used flattened cortical surface representations of each subject's occipital and parietal cortices to visualize amplitude, coherence, and phase maps. Visual area boundaries were drawn on retinotopic maps based on standard conventions (Larsson and Heeger, 2006; Wandell et al., 2007). Although we acknowledge that there is some controversy over the definition of area hV4 (Brewer et al., 2005), we defined area hV4 according to the convention of Brewer et al. (2005) and Wandell et al. (2007). For the localizer data, we used the same approach. For each visual area, we used the localizer maps to define two ROIs in each hemisphere, one corresponding to the upper quadrant and one to the lower quadrant of the visual field. Each quadrant ROI, therefore, contained voxels that responded to the locations of two of the saccade targets (e.g., upper right). For a few subjects, we had difficulties defining a quadrant of hV4 or VO1, possibly due to artifacts from the transverse sinus (Winawer et al., 2010). We could not reliably define one quadrant of hV4 in two subjects, and one quadrant of VO1 in three subjects ( 2 left and 1 right upper VF). For these quadrants, we restricted our analyses to the remaining subjects.

Measuring delay period activity. Our overall goal was to measure the spatial specificity of blood oxygen-level-dependent (BOLD) responses across the retinotopic ROIs during the delay period of memory-guided saccade tasks. We estimated (1) the time courses of activation for each trial type and (2) parameter fits of response amplitudes specific to the target, delay, and response periods. For each subject, saccade task, and visual area, the fMRI response time courses were segregated and pooled separately for each quadrant ROI. A global response component has been reported previously in the visual cortex during different visual tasks and phenomena including detection, discrimination, and motion-induced blindness (Jack et al., 2006; Donner et al., 2008), and is believed to be nonsensory and spatially nonspecific. We assumed that this global, spatially nonspecific component is superimposed with the stimulus specific component we were interested in measuring. Indeed, spontaneous neural fluctuations (Leopold and Logothetis, 2003) superimpose linearly with stimulus induced responses (Fox et al., 2006), and spatially specific estimation improves when these fluctuations are modeled, especially when the task structure can be anticipated by the subject (Jack et al., 2006; Sylvester et al., 2007). Therefore, to improve the spatial specificity of our estimated responses in visual cortex, within each ROI time course, we projected out a global, spatially nonspecific nuisance component from control ROIs in anterior calcarine sulcus in each hemisphere representing the far peripheral $\left(20^{\circ}-40^{\circ}\right)$ visual field. A residual task-specific time series was computed for each voxel in the quadrant ROI with orthogonal projection, $y^{*}=y-\left(y^{T} r\right) r$, where $y$ was the original time series of the quadrant ROI, $r$ was the unit vector reference time series, and $y^{*}$ was the residual, task-specific time series. By removing $\mathrm{r}$ using orthogonal projection, we ensured that the exact amount of $r$ present in $y$ was removed. We trial averaged the resulting time courses time locked to the visual target onset. We truncated the averaging at the end of the delay to prevent the saccade-evoked response contaminating the delay period estimation. We used multiple linear regressions to estimate the responses to the target, delay, and response intervals. The target and response intervals were modeled with transient neural events. The delay period was modeled by a combination of two orthogonal covariates, a zero-order and a first-order polynomial, to model both steps and linear trends in delayperiod activity. Each covariate was convolved with a canonical model of the hemodynamic impulse response function, linearly detrended and high-pass filtered in the same way as the measured fMRI data. This model was not applied on a voxelwise basis across the brain, as traditionally done. Instead, for each subject we created one time series for each ROI by averaging the BOLD signal from each voxel within each quadrant ROI across all runs (Schluppeck et al., 2006; Offen et al., 2009). Critically, the design matrix modeled each covariate as a function of the saccade goal's quadrant location (in, adjacent, mirror, diagonal; Fig. 1D), so the topographic specificity of the responses could be precisely estimated and compared.

To compare the model with the observed fMRI responses, we calculated the mean and the SEM of the model fit using the same trial- averaging analysis described above. To fit the model, we multiplied the $\beta$ weights for each covariate by the design matrix, which had been convolved and filtered, and encoded the quadrant trial types (Schluppeck et al., 2006; Offen et al., 2009; see Materials and Methods). The result, when concatenated across trial epochs within each of the four quadrant ROIs, was a time course the length of the observed data, which was trial averaged (plus SEM) exactly like the observed data. To quantify the goodness-of-fit of the model, we computed the proportion of variance accounted for in the observed trial averaged data $\left(r^{2}\right)$, where $r^{2}=1-$ $\left(\sigma_{\text {residual }}^{2} / \sigma_{\text {observed }}^{2}\right)$, and residuals $=($ model - data $)$.

Statistical analyses. We used permutation tests to estimate statistical significances (Larsson and Heeger, 2006; Pestilli et al., 2011). For each subject, saccade task, and visual area, we tested whether delay period responses: (1) were significantly different from zero, and (2) were significantly different across trial types. The permutation test compared the estimated delay period response with a distribution of responses expected by chance according to the null hypotheses that: (1) there was no delay-period activity, and (2) there was no difference between evoked delay-period activity across trial types (i.e., delay-period activity was not spatially specific). We generated 10,000 samples for the statistical distributions of the two null hypotheses. In each iteration of the permutation test, we generated a shuffled trial sequence for all trials and repeated the linear regression described above to generate null distributions and compute the probabilities ( $p$ values) of observing the differences in our actual data.

\section{Results}

\section{Similar response times and targeting errors for prosaccades} and antisaccades

Overall, $85 \%$ and $91 \%$ of all memory-guided prosaccade and antisaccade response times (SRTs) fell within the normal range of visually guided saccades (i.e., 200-500 ms; Fig. 1B, left), and differences in SRTs between the two tasks were not significant ( $p>0.05$, permutation test; Fig. $1 B$, inset). In addition, primary saccade error was indistinguishable between prosaccades and antisaccades ( $p>0.05$, permutation test; Fig. $1 B$, right). Often during memory-guided saccades, subjects adjust their gaze slightly after the primary saccade but before feedback, which typically reduces overall error. Indeed, secondary saccades reduced error in both tasks (mean $\pm \mathrm{SEM}=1.3^{\circ} \pm 0.2^{\circ}$ prosaccade; $1.4^{\circ}$ $\pm 0.2^{\circ}$ antisaccade). Critically, average errors after secondary saccades were not significantly different between the two tasks $(p>$ 0.05 , permutation test; Fig. $1 B$ ). Peak velocities were also indistinguishable between the two types of delayed saccades (mean \pm $\mathrm{SEM}=432 \pm 29 \%$ prosaccade; $448 \pm 36 \%$ antisaccade; $p>$ 0.05 , permutation test). Therefore, during the delay period subjects planned prosaccades and antisaccades with similar timing and speed to the same spatial location with similar precision. Such remarkably similar performance for prosaccades and antisaccades can be accounted for by the fact that in both tasks subjects had enough time during the delay period to plan an eye movement to the appropriate saccade goal.

\section{The maintenance of a saccade plan elicits topographically specific persistent activity in early visual areas in the dorsal and ventral streams}

We asked whether planning memory-guided saccades would elicit persistent cortical activity in the retinotopic location of visual maps matching the saccade goal location. To answer this question, we used retinotopic mapping (Wandell et al., 2007; Pestilli et al., 2011) to identify separate visual maps along the dorsal and ventral stream. We identified early visual areas V1, V2, and V3, dorsal stream visual areas V3A/B and IPS0, and ventral stream visual areas hV4 and VO1 (Figs. 1C). Within each one of these retinotopic maps, we localized the cortical subregion cor- 

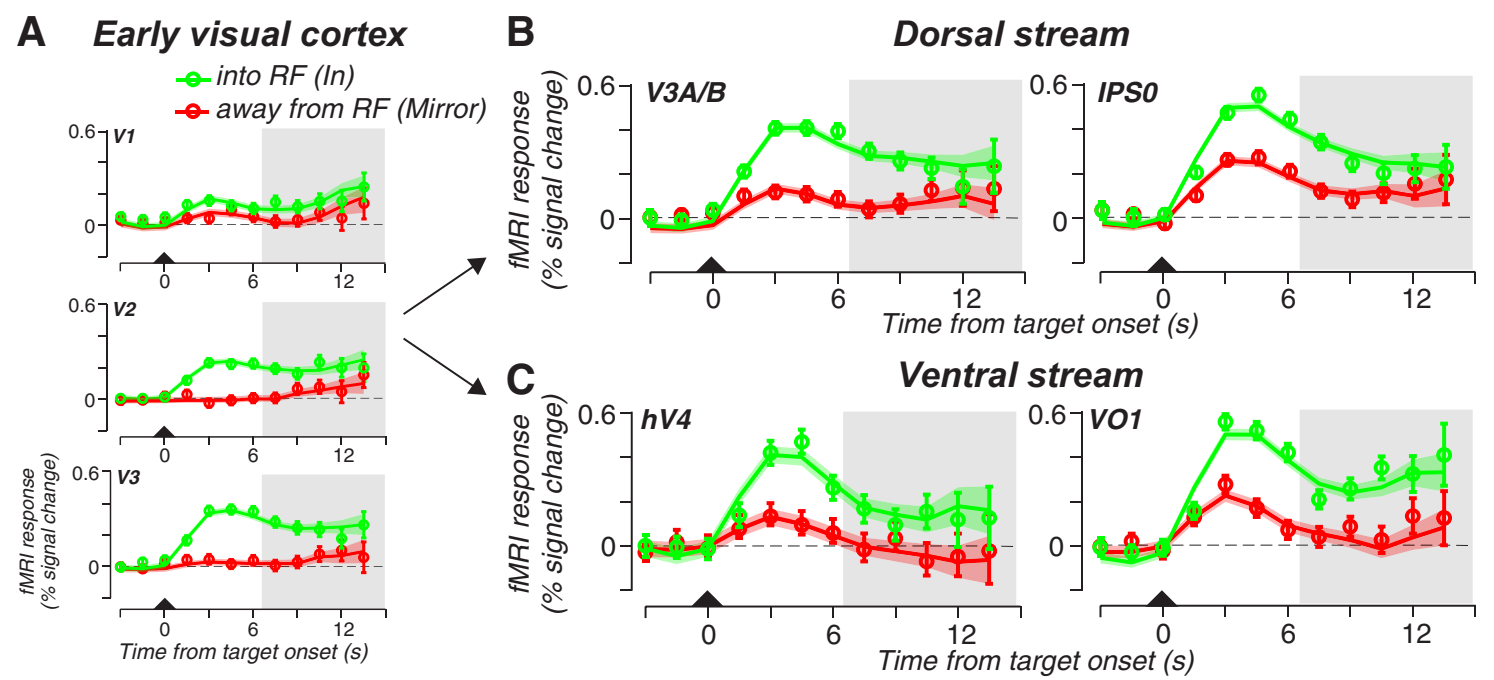

Figure 2. Trial averaged time courses during the maintenance of planned prosaccades. $A$, Following a transient visual response to the brief visual target (triangle), activity persisted in early visual areas in the part of the retinotopic map that represented the location of the visual target and matching saccade goal (saccade plan into RF, green) but not in a control area that mapped the mirror location in the opposite hemifield (saccade plan away from RF, mirror, red). $\boldsymbol{B}$, In the dorsal stream retinotopic ROIs, V3A/B and IPSO, spatially specific activity persisted when the visual target and planned saccade fell within the ROI's mapped RF quadrant. C, Similarly, activity persisted in ventral stream ROIs, hV4 and V01, when the visual target and saccade goal matched the ROI's mapped RF quadrant. Shaded area bounds the delay-period activity. Open circles represent the mean fMRI response across trials and subjects. Error bars represent SEM. The solid line and error ribbon represent the mean and SEM from the model fits (see Materials and Methods). Model fits the observed data well; $r^{2}{ }_{\text {IN }}=0.85 ; r^{2}{ }_{\text {MIRROR }}=0.80$.

responding to a $2^{\circ}$ wide arc centered at an eccentricity of $10^{\circ}$ within each quarterfield (Fig. $1 C$; Pestilli et al., 2011). Visual targets and saccade endpoints (Fig. $1 B$, right) fell within these $2^{\circ}$ arcs in each visual quadrant.

Cortical activity was measured at the retinotopic locations of prosaccades and antisaccades endpoints as defined above. We used an event-related memory-guided saccade task, and for each trial in each visual area, we extracted fMRI responses from the retinotopic ROI categorized according to the quadrant into which the saccade was planned. Trials in which the saccade goal was mapped to the receptive field (RF) of the ROI were labeled as "In." Trials in which the saccade goal was not mapped to the ROI's RF were divided into three groups depending on whether the quadrant was adjacent to, the mirror of, or diagonal to the ROI's RF (Fig. 1D).

We first consider activity in early visual areas V1-V3 during the maintenance of memory-guided prosaccades. Activity limited to the portions of the retinotopic maps that matched the endpoint of the saccade plan persisted above pretrial baseline and above the other locations in the maps (Fig. $2 A$, shaded area). In addition to early visual cortex, we mapped four other visual field maps along the dorsal and ventral visual streams, V3A/B, IPS0, and hV4, VO1, respectively. Spatially specific delay-period activity persisted in both dorsal and ventral visual field maps (Fig. $2 B, C)$. We used regression analyses to estimate the magnitude of persistent delay-period activity after accounting for transient responses to visual targets and memory-guided saccades (Schluppeck et al., 2006; Offen et al., 2009; see Materials and Methods). Consistent with the time courses in Figure 2, delay-period activity was greatest at the location that matched the saccade goal $(p<$ 0.005, permutation test; Fig. 3).

These results demonstrate spatially specific persistent activity in early visual cortex and along both dorsal and ventral visual streams. Ideally, we would like to attribute this response to a top-down process related to the goal of the planned saccade. Methodologically, however, our regression analyses best partition the signal variance between the trial components, but does assume a linear transfer from neural activity to measured hemodynamic response. Thus, the visual response to the saccade target might contaminate our delay period estimates despite our efforts to disambiguate the two responses (i.e., long and variable delays and partial trials to break the temporal contingency). The responses could reflect a nonlinear or lingering trace of the bottom-up visual target. Theoretically, we aimed to test whether saccade planning in the absence of bottom-up stimulation could elicit delay-period activity. To address these potential methodological and theoretical concerns, we next used an antisaccade task that dissociated the location of the visual target from the mirrored saccade goal. This allowed us to test critical hypotheses about the nature of the persistent activity.

\section{Saccade planning initiates spatially selective persistent activity in the absence of visual stimulation}

FEF microstimulation has been shown to enhance the firing rates of V4 neurons only when a visual stimulus is concurrently presented within its RF (Moore and Armstrong, 2003). Similarly, FEF microstimulation causes BOLD signal increases in retinotopic areas that match the FEF neuron's RF only when accompanied by concurrent visual stimulation (Ekstrom et al., 2008). These findings suggest that the effectiveness of top-down signals depend on, or are gated by, bottom-up stimulation.

To test whether the presence of a stimulus is necessary for eliciting delay-period activity, we measured cortical activity during planned antisaccades in the portions of the retinotopic maps that matched the endpoint of the saccade plan, and not that of the visual target. We found strong increases in cortical activity during the delay period at the retinotopic portion of each map representing the antisaccade goal (Fig. 4, green). The dynamics of the time courses were uncharacteristic of the brief phasic nature of responses to attended visual targets (Liu et al., 2005; Pestilli et al., 2011). Indeed, the time courses from ROIs that map the location of the saccade goal lack the large transient response time locked to the visual target (Fig. 4, green). Instead, the response ramped up only after the antisaccade to the mirror location had been 


\section{A Early visual cortex}

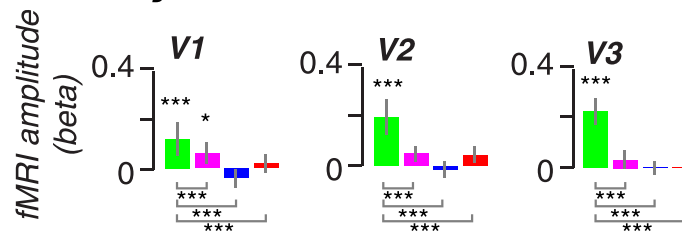

B Dorsal stream

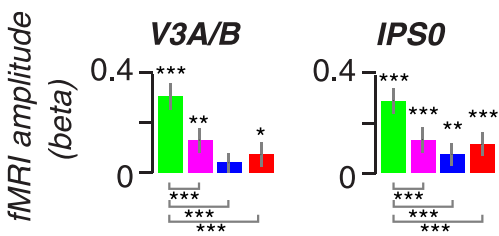

C Ventral stream

In $\square$ Adjacent $\square$ Diagonal $\square$ Mirror

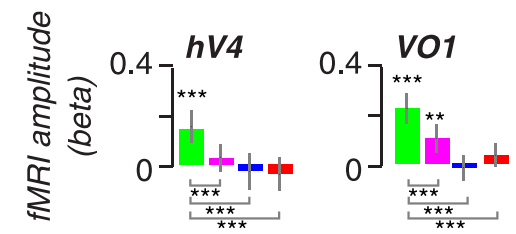

Figure 3. Cortical responses during the maintenance of planned prosaccades. Memory-guided saccade responses in $(\boldsymbol{A})$ early visual cortex, $(\boldsymbol{B})$ the dorsal stream, and $(\boldsymbol{C})$ the ventral stream. Each panel depicts modeled amplitude of delay period activity for prosaccades for each visual area. Responses are ordered by trial type according to the location of the topographic map matching the planned saccade (Fig. 1 D). Green bars (In) show responses from voxels that map the goal of the planned saccade. Red bars (Mirror) show responses that map the mirror quadrant of the goal of the planned saccade. Magenta and blue bars show responses from the adjacent and diagonal quadrants, respectively, of the planned saccade. Each bar represents the mean amplitude of the fitted delay period response across subjects ( \pm SEM). Asterisks indicate significant delay period activity more than zero (above bars) and significant activity differences between when the saccade plan was into the RF versus the other locations (below bars), where ${ }^{*} p<0.05,{ }^{* *} p<0.01$, and ${ }^{* * *} p<0.005$, determined by permutation tests.

A Early visual cortex - into RF (In) $\rightarrow$ away from RF (Mirror)
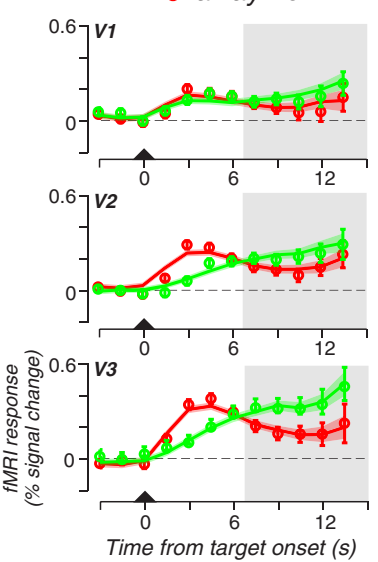

B

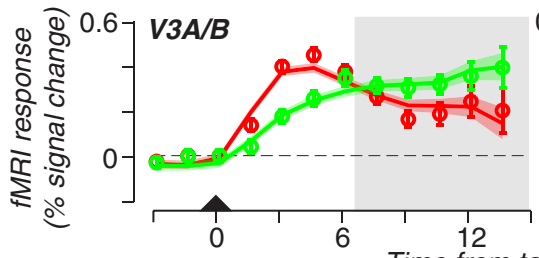

Time from target onset (s)
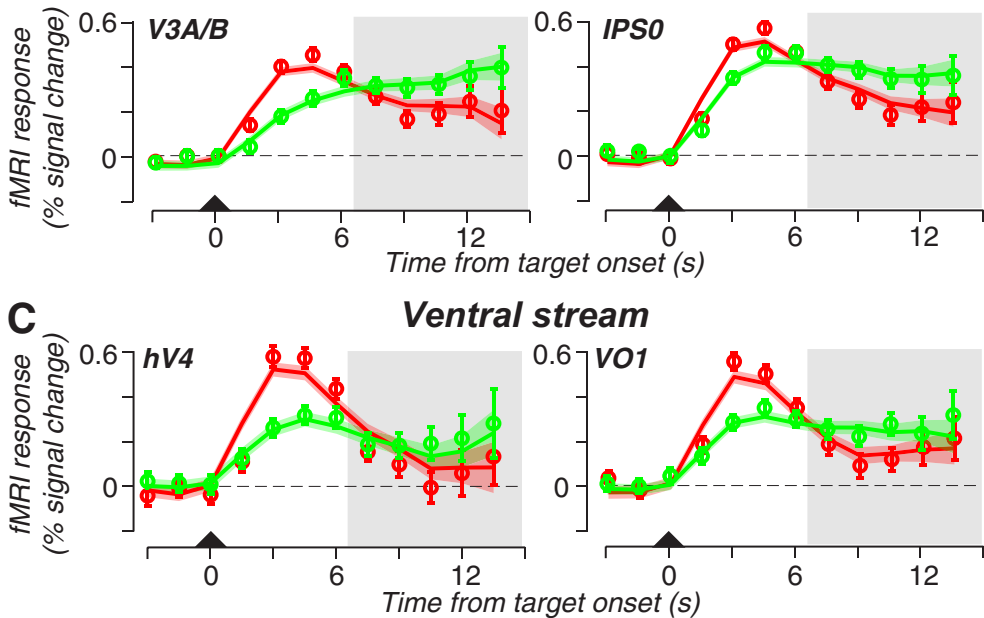

Figure 4. Trial averaged time courses during the maintenance of planned antisaccades. $\boldsymbol{A}$, First, the brief visual target (triangle) evoked a transient response in early visual areas in the part of the retinotopic map that represented the location of the visual target similar to planned prosaccades (Mirror RF to the saccade plan, red). Then, activity ramped up and persisted in the part of the retinotopic map that represented the saccade goal (saccade plan into RF, green). $\boldsymbol{B}$, In the dorsal stream retinotopic R0Is, V3A/B and IPSO, spatially specific activity persisted when the planned saccade fell within the ROI's mapped RF quadrant. C, Similarly, activity persisted in ventral stream ROIs, hV4 and V01, when the saccade goal matched the ROI's mapped RF quadrant. Shaded area bounds the delay-period activity. Open circles represent the mean $\mathrm{fMRI}$ response across trials and subjects. Error bars represent SEM. The solid line and error ribbon represents the mean and SEM from the model fits (see Materials and Methods). Model fits the observed data well; $r^{2}{ }_{\text {IN }}=0.85 ; r^{2}{ }_{\text {MIRROR }}=0.87$.

planned. Motor preparation evokes precisely this type of ramping dynamics (Mars et al., 2008). Interestingly, we also measured a robust early response in the ROIs mapping the location of the attended visual target that persisted with smaller magnitude during the delay (Fig. 4, red). Consistent with the time courses in Figure 4, our regression analyses confirmed that the mean delayperiod activity was greatest at the location that matched the saccade goal ( $p<0.005$, permutation test; Fig. 5$)$. We attribute these retinotopically specific delay-period activities to top-down signals evoked by saccade planning.

\section{Discussion}

Our results support the hypothesis that activity persists in the specific parts of retinotopic maps that represent the location of saccade goals. We find that topographically specific activity persisted in early visual areas (V1, V2, and V3) and also in higher order visual areas in both the dorsal stream (V3A/B, IPS0) and the ventral stream (hV4, VO1). Our findings are consistent with an early fMRI blocked design study that reported activity, which was visually evoked by high-contrast flashing checkerboards during a memory delay, was greater in the occipital hemisphere contralateral to the visual field that once contained memorized stimuli (Awh et al., 1999). Unlike the only other previous study of the effects of saccade planning on human visual areas (Geng et al., 2009), we used an event-related design that avoided the assumptions of subtractive logic and allowed us to directly measure activity in precisely defined retinotopic areas resulting from top- 


\section{A Early visual cortex}

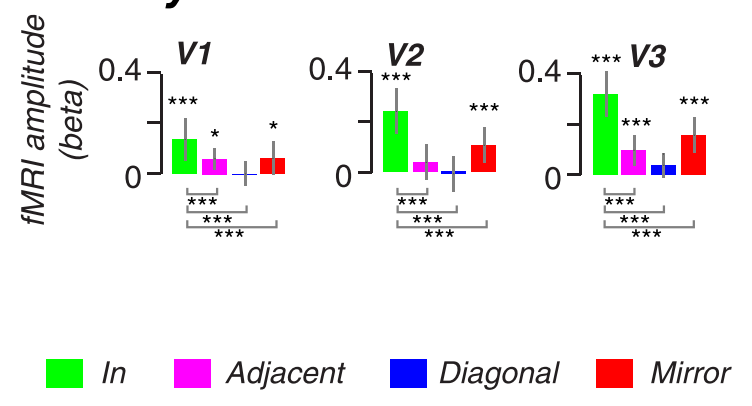

\section{B Dorsal stream}

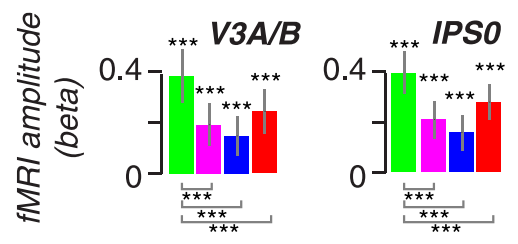

C Ventral stream

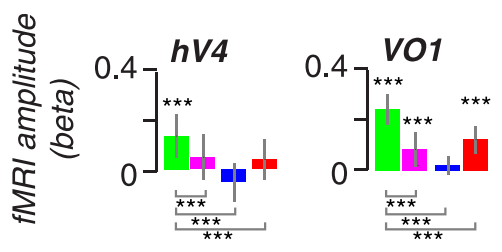

Figure 5. Cortical responses during the maintenance of planned antisaccades. Memory-guided saccade responses in $(\boldsymbol{A})$ early visual cortex, $(\boldsymbol{B})$ the dorsal stream, and $(\boldsymbol{C})$ the ventral stream. Each panel depicts modeled amplitude of delay period activity for antisaccades for each visual area. Responses are ordered by trial type according to the location of the topographic map matching the planned saccade (Fig. 1D). Green bars (In) show responses from voxels that map the goal of the planned saccade. Red bars (Mirror) show responses that map the mirror quadrant of the goal of the planned saccade, which also correspond to the visual saccade target's location. Magenta and blue bars show responses from the adjacent and diagonal quadrants, respectively, of the planned saccade. Each bar represents the mean amplitude of the fitted delay period response across subjects ( \pm SEM). Asterisks indicate significant delay period activity more than zero (above bars) and significant activity differences between when the saccade plan was into the RF versus the other locations (below bars), where ${ }^{*} p<0.05,{ }^{* *} p<0.01$, and ${ }^{* * *} p<0.005$, determined by permutation tests.

down signals uncontaminated by bottom-up sensory stimulation and saccade execution. We found spatially selective activity that persisted throughout the memory delay. Surprisingly, previous human studies have failed to find reliable persistent activity in visual cortex during delays in which subjects maintained feature information (Ester et al., 2009; Harrison and Tong, 2009; Offen et al., 2009; Serences et al., 2009; Riggall and Postle, 2012; Albers et al., 2013), but the same studies have instead shown that the contents (e.g., orientation, color, spatial frequency, motion) of working memory can be decoded from the multivoxel patterns of delay-period activity in visual cortex. Together, these results lead to many open questions about the role of spatially organized top-down signals in the maintenance of retinotopic featurespecific representations (Sreenivasan et al., 2014; Pratte and Tong, 2014). The fact that we measured robust spatially selective persistent activity during the memory delay is theoretically critical. Compared with multivoxel decoding, where the neural mechanisms remain unknown and certainly controversial (Freeman et al., 2011; Xing et al., 2013), the mechanisms of persistent activity have been well studied and modeled for decades (Fuster and Alexander, 1971; Funahashi et al., 1989; Wang, 2001; Curtis and D'Esposito, 2003; Major and Tank, 2004; Sreenivasan et al., 2014). Our findings are consistent with the sensory-recruitment model of working memory, which posits that working memory is supported by the same neural mechanisms used to encode the sensory information to be maintained (Pasternak and Greenlee, 2005; Postle, 2006). Specifically here, the spatial goal might be maintained in retinotopic visual cortex through a feedback signal initiated in frontal cortex that induces a spatially selective increase in neural activity that persists until the memory-guided response is initiated.

Although the mechanisms of top-down control remain nebulous, theoretical and experimental evidence favors feedback in the form of saccade planning signals. Oculomotor signals share a spatial coordinate framework with retinotopically organized visual areas. Psychophysical studies indicate that saccade planning and attention are somehow intertwined (Rizzolatti et al., 1987;
Hoffman and Subramaniam, 1995; Kowler et al., 1995; Deubel and Schneider, 1996). For instance, visual sensitivity and perceived contrast are better at the goal of a planned saccade (Rolfs et al., 2011). Critically, microstimulation of monkey FEF neurons, at levels too low to evoke a saccade, improves visual performance (Moore and Fallah, 2004) and enhances visual responses in V4 at locations that match the endpoints of saccades that would be evoked with greater current (Moore and Armstrong, 2003). Moreover, activity persists in monkey V1 neurons with RFs that specifically match the goal of a memory-guided saccade (Supèr et al., 2001). The present results provide strong support for the model that the readout of oculomotor signals by visual cortex prioritize locations matching the saccade goal via gain changes in neural excitability.

We measured brain activity and saccades in human subjects who were highly trained to plan and execute accurate memoryguided saccades. We used two independent saccade conditions that allowed us to measure the effects of covert attention and saccade planning at separate retinal locations. A similar logic was used to dissociate spatial attention and saccade preparation in FEF (Juan et al., 2004), and memory and attention in dorsolateral prefrontal cortex (Messinger et al., 2009). We found early transient responses time-locked to the presentation of the saccade target in both prosaccade and antisaccade trials that mapped to the retinotopic location of the attended visual target, consistent with previous studies of the effects of attention on visual cortex (Pestilli et al., 2011). The behaviorally relevant visual transient attracted covert attention and evoked activity in the parts of visual cortex that mapped the location of the visual stimulus. In the antisaccade condition, we found an additional ramping response during the delay in parts of visual cortex that mapped the location of the saccade plan, not the covertly attended visual target. Such linear dynamics closely match the canonical ramping response associated with motor preparation (Mars et al., 2008).

Unlike the effects of saccade planning, the effects of covert attention on macaque visual cortex have been documented and reviewed extensively (Reynolds and Chelazzi, 2004; Reynolds and 
Heeger, 2009). Despite a relative lack of experimental evidence, many have assumed that the effects of covert and overt attention on visual cortex may be the same (Moore et al., 2003). A recent study demonstrated that planning a saccade to an irrelevant target, while covertly attending a relevant target, caused indistinguishably enhanced activity in macaque V4 neurons with RFs that matched the saccade and attended targets (Steinmetz and Moore, 2014). Therefore, saccade preparation is sufficient to modulate the neuronal activity of macaque visual neurons. Our data support these findings from macaque V4 and indicate a striking similarity between the species even when using very different measurement techniques. Importantly, we demonstrate that saccade planning enhances activity not only in V4, but also along the entire visual hierarchy.

Results from studies using animal models of human cognition suggest that top-down signals are gated by, or require the simultaneous presence of bottom-up visual stimulation. For instance, microstimulation of monkey FEF, which is thought to simulate top-down signals associated with saccade planning, does not evoke spatially specific BOLD activity in retinotopic visual cortex unless stimulation is paired with a visual stimulus (Ekstrom et al., 2008). We find persistent activity in retinotopic visual areas that map the saccade goal when the visual target was only available via working memory, even when that location was not visually stimulated before the memory delay. Therefore, maintenance of the saccade goal is sufficient to instantiate persistent activity. This is not surprising given that covert attention, a related top-down process, also elicits retinotopically specific neural activity in early visual cortex in the absence of visual stimulation (Kastner et al., 1999). Perhaps the nature of the top-down signal could be causing the inconsistent results; brief microstimulation might not evoke as large of a BOLD response as long epochs of saccade preparation. In aggregate, it appears as if spatially specific topdown signals caused by saccade planning, attention, and working memory may each effect activity in early visual cortex similarly, despite that these spatial cognitive abilities may have distinct neuronal origins (Juan et al., 2004; Messinger et al., 2009; Suzuki and Gottlieb, 2013). Future work should target whether these effects and their functional consequences are dissociable at the level of the visual cortex.

Topographically specific delay-period activity has been previously demonstrated within visual maps along the dorsal stream (Schluppeck et al., 2006; Jerde et al., 2012), in areas which code for spatial and visuomotor control (Goodale and Milner, 1992; Ungerleider and Haxby, 1994), but not in early visual cortex (Offen et al., 2009). Here, we show that similar delay-period signals exist in early visual cortex (V1, V2, V3) and are stronger in the early dorsal stream. Activity persisted at the saccade-goal representation in early visual cortex (V1, V2, V3) and in the dorsal stream (V3A/B and IPS0) areas involved in spatial orienting and saccade preparation (Schluppeck et al., 2005; Silver et al., 2005; Kastner et al., 2007; Swisher et al., 2007; McKeefry et al., 2008; Fischer et al., 2012; Jerde et al., 2012). Furthermore, we show for the first time in the human brain that saccade planning elicits spatially specific persistent activity also in ventral visual areas (hV4 and VO1) traditionally related primarily to object, features, and form processing (Bartels and Zeki, 2000; Wade et al., 2002; Brewer et al., 2005; Arcaro et al., 2009). These spatial feedback signals may bias the competition for neural representation in favor of feature and object selective neurons with RFs that overlap the location of the saccade goal (Jerde et al., 2012).

We conclude that the effects of spatially specific top-down signals activated during saccade preparation elicit corresponding activity in early retinotopic visual areas and along the dorsal and ventral visual streams. Such a gain enhancement might underlie the mechanisms that prioritize the locations of task-relevant items in visual space.

\section{References}

Albers AM, Kok P, Toni I, Dijkerman HC, de Lange FP (2013) Shared representations for working memory and mental imagery in early visual cortex. Curr Biol 23:1427-1431. CrossRef Medline

Arcaro MJ, McMains SA, Singer BD, Kastner S (2009) Retinotopic organization of human ventral visual cortex. J Neurosci 29:10638-10652. CrossRef Medline

Awh E, Jonides J (2001) Overlapping mechanisms of attention and spatial working memory. Trends Cogn Sci 5:119-126. CrossRef Medline

Awh E, Jonides J, Smith EE, Buxton RB, Frank LR, Love T, Wong EC, Gmeindl L (1999) Rehearsal in spatial working memory: evidence from neuroimaging. Psychol Sci 10:433-437. CrossRef

Awh E, Armstrong KM, Moore T (2006) Visual and oculomotor selection: links, causes and implications for spatial attention. Trends Cogn Sci 10: 124-130. CrossRef Medline

Bartels A, Zeki S (2000) The architecture of the colour centre in the human visual brain: new results and a review. Eur J Neurosci 12:172-193. CrossRef Medline

Brewer AA, Liu J, Wade AR, Wandell BA (2005) Visual field maps and stimulus selectivity in human ventral occipital cortex. Nat Neurosci 8:11021109. CrossRef Medline

Corbetta M, Kincade JM, Shulman GL (2002) Neural systems for visual orienting and their relationships to spatial working memory. J Cogn Neurosci 14:508-523. CrossRef Medline

Curtis CE, Connolly JD (2008) Saccade preparation signals in the human frontal and parietal cortices. J Neurophysiol 99:133-145. CrossRef Medline

Curtis CE, D'Esposito M (2003) Persistent activity in the prefrontal cortex during working memory. Trends Cogn Sci 7:415-423. CrossRef Medline

Desimone R, Duncan J (1995) Neural mechanisms of selective visual attention. Annu Rev Neurosci 18:193-222. CrossRef Medline

Deubel H, Schneider WX (1996) Saccade target selection and object recognition: evidence for a common attentional mechanism. Vision Res 36: 1827-1837. CrossRef Medline

Donner TH, Sagi D, Bonneh YS, Heeger DJ (2008) Opposite neural signatures of motion-induced blindness in human dorsal and ventral visual cortex. J Neurosci 28:10298-10310. CrossRef Medline

Ekstrom LB, Roelfsema PR, Arsenault JT, Bonmassar G, Vanduffel W (2008) Bottom-up dependent gating of frontal signals in early visual cortex. Science 321:414-417. CrossRef Medline

Ester EF, Serences JT, Awh E (2009) Spatially global representations in human primary visual cortex during working memory maintenance. J Neurosci 29:15258-15265. CrossRef Medline

Fischer E, Bülthoff HH, Logothetis NK, Bartels A (2012) Human areas V3A and V6 compensate for self-induced planar visual motion. Neuron 73: 1228-1240. CrossRef Medline

Fox MD, Snyder AZ, Zacks JM, Raichle ME (2006) Coherent spontaneous activity accounts for trial-to-trial variability in human evoked brain responses. Nat Neurosci 9:23-25. CrossRef Medline

Freeman J, Brouwer GJ, Heeger DJ, Merriam EP (2011) Orientation decoding depends on maps, not columns. J Neurosci 31:4792-4804. CrossRef Medline

Funahashi S, Bruce CJ, Goldman-Rakic PS (1989) Mnemonic coding of visual space in the monkey's dorsolateral prefrontal cortex. J Neurophysiol 61:331-349. Medline

Fuster JM, Alexander GE (1971) Neuron activity related to short-term memory. Science 173:652-654. CrossRef Medline

Geng JJ, Ruff CC, Driver J (2009) Saccades to a remembered location elicit spatially specific activation in human retinotopic visual cortex. J Cogn Neurosci 21:230-245. CrossRef Medline

Goodale MA, Milner AD (1992) Separate visual pathways for perception and action. Trends Neurosci 15:20-25. CrossRef Medline

Harrison SA, Tong F (2009) Decoding reveals the contents of visual working memory in early visual areas. Nature 458:632-635. CrossRef Medline

Hoffman JE, Subramaniam B (1995) The role of visual attention in saccadic eye movements. Percept Psychophys 57:787-795. CrossRef Medline

Jack AI, Shulman GL, Snyder AZ, McAvoy M, Corbetta M (2006) Separate 
modulations of human V1 associated with spatial attention and task structure. Neuron 51:135-147. CrossRef Medline

Jerde TA, Merriam EP, Riggall AC, Hedges JH, Curtis CE (2012) Prioritized maps of space in human frontoparietal cortex. J Neurosci 32:1738217390. CrossRef Medline

Juan CH, Shorter-Jacobi SM, Schall JD (2004) Dissociation of spatial attention and saccade preparation. Proc Natl Acad Sci U S A 101:15541-15544. CrossRef Medline

Kastner S, Pinsk MA, De Weerd P, Desimone R, Ungerleider LG (1999) Increased activity in human visual cortex during directed attention in the absence of visual stimulation. Neuron 22:751-761. CrossRef Medline

Kastner S, DeSimone K, Konen CS, Szczepanski SM, Weiner KS, Schneider KA (2007) Topographic maps in human frontal cortex revealed in memory-guided saccade and spatial working-memory tasks. J Neurophysiol 97:3494-3507. CrossRef Medline

Kowler E, Anderson E, Dosher B, Blaser E (1995) The role of attention in the programming of saccades. Vision Res 35:1897-1916. CrossRef Medline

Larsson J, Heeger DJ (2006) Two retinotopic visual areas in human lateral occipital cortex. J Neurosci 26:13128-13142. CrossRef Medline

Leopold DA, Logothetis NK (2003) Spatial patterns of spontaneous local field activity in the monkey visual cortex. Rev Neurosci 14:195-205. Medline

Liu T, Pestilli F, Carrasco M (2005) Transient attention enhances perceptual performance and FMRI response in human visual cortex. Neuron 45: 469-477. CrossRef Medline

Major G, Tank D (2004) Persistent neural activity: prevalence and mechanisms. Curr Opin Neurobiol 14:675-684. CrossRef Medline

Mars RB, Coles MG, Hulstijn W, Toni I (2008) Delay-related cerebral activity and motor preparation. Cortex 44:507-520. CrossRef Medline

McKeefry DJ, Burton MP, Vakrou C, Barrett BT, Morland AB (2008) Induced deficits in speed perception by transcranial magnetic stimulation of human cortical areas V5/MT+ and V3A. J Neurosci 28:6848-6857. CrossRef Medline

Messinger A, Lebedev MA, Kralik JD, Wise SP (2009) Multitasking of attention and memory functions in the primate prefrontal cortex. J Neurosci 29:5640-5653. CrossRef Medline

Moore T, Armstrong KM (2003) Selective gating of visual signals by microstimulation of frontal cortex. Nature 421:370-373. CrossRef Medline

Moore T, Fallah M (2004) Microstimulation of the frontal eye field and its effects on covert spatial attention. J Neurophysiol 91:152-162. CrossRef Medline

Moore T, Armstrong KM, Fallah M (2003) Visuomotor origins of covert spatial attention. Neuron 40:671-683. CrossRef Medline

Offen S, Schluppeck D, Heeger DJ (2009) The role of early visual cortex in visual short-term memory and visual attention. Vision Res 49:1352-1362. CrossRef Medline

Pasternak T, Greenlee MW (2005) Working memory in primate sensory systems. Nat Rev Neurosci 6:97-107. CrossRef Medline

Pestilli F, Carrasco M, Heeger DJ, Gardner JL (2011) Attentional enhancement via selection and pooling of early sensory responses in human visual cortex. Neuron 72:832-846. CrossRef Medline

Postle BR (2006) Working memory as an emergent property of the mind and brain. Neuroscience 139:23-38. CrossRef Medline

Pratte MS, Tong F (2014) Spatial specificity of working memory representations in the early visual cortex. J Vis 14(3):22 1-12. CrossRef Medline

Reynolds JH, Chelazzi L (2004) Attentional modulation of visual processing. Annu Rev Neurosci 27:611-647. CrossRef Medline

Reynolds JH, Heeger DJ (2009) The normalization model of attention. Neuron 61:168-185. CrossRef Medline
Riggall AC, Postle BR (2012) The relationship between working memory storage and elevated activity as measured with functional magnetic resonance imaging. J Neurosci 32:12990-12998. CrossRef Medline

Rizzolatti G, Riggio L, Dascola I, Umiltá C (1987) Reorienting attention across the horizontal and vertical meridians: evidence in favor of a premotor theory of attention. Neuropsychologia 25:31-40. CrossRef Medline

Rolfs M, Jonikaitis D, Deubel H, Cavanagh P (2011) Predictive remapping of attention across eye movements. Nat Neurosci 14:252-256. CrossRef Medline

Schluppeck D, Glimcher P, Heeger DJ (2005) Topographic organization for delayed saccades in human posterior parietal cortex. J Neurophysiol 94: 1372-1384. CrossRef Medline

Schluppeck D, Curtis CE, Glimcher PW, Heeger DJ (2006) Sustained activity in topographic areas of human posterior parietal cortex during memory-guided saccades. J Neurosci 26:5098-5108. CrossRef Medline

Serences JT, Ester EF, Vogel EK, Awh E (2009) Stimulus-specific delay activity in human primary visual cortex. Psychol Sci 20:207-214. CrossRef Medline

Silver MA, Ress D, Heeger DJ (2005) Topographic maps of visual spatial attention in human parietal cortex. J Neurophysiol 94:1358-1371. CrossRef Medline

Sreenivasan KK, Curtis CE, D’Esposito M (2014) Revisiting the role of persistent neural activity during working memory. Trends Cogn Sci 18:8289. CrossRef Medline

Srimal R, Curtis CE (2008) Persistent neural activity during the maintenance of spatial position in working memory. Neuroimage 39:455-468. CrossRef Medline

Steinmetz NA, Moore T (2014) Eye movement preparation modulates neuronal responses in area $\mathrm{v} 4$ when dissociated from attentional demands. Neuron 83:496-506. CrossRef Medline

Supèr H, Spekreijse H, Lamme VA (2001) A neural correlate of working memory in the monkey primary visual cortex. Science 293:120-124. CrossRef Medline

Suzuki M, Gottlieb J (2013) Distinct neural mechanisms of distractor suppression in the frontal and parietal lobe. Nat Neurosci 16:98-104. CrossRef Medline

Swisher JD, Halko MA, Merabet LB, McMains SA, Somers DC (2007) Visual topography of human intraparietal sulcus. J Neurosci 27:5326-5337. CrossRef Medline

Sylvester CM, Shulman GL, Jack AI, Corbetta M (2007) Asymmetry of anticipatory activity in visual cortex predicts the locus of attention and perception. J Neurosci 27:14424-14433. CrossRef Medline

Ungerleider LG, Haxby JV (1994) "What" and "where" in the human brain. Curr Opin Neurobiol 4:157-165. CrossRef Medline

Wade AR, Brewer AA, Rieger JW, Wandell BA (2002) Functional measurements of human ventral occipital cortex: retinotopy and colour. Philos Trans R Soc Lond B Biol Sci 357:963-973. CrossRef Medline

Wandell BA, Dumoulin SO, Brewer AA (2007) Visual field maps in human cortex. Neuron 56:366-383. CrossRef Medline

Wang XJ (2001) Synaptic reverberation underlying mnemonic persistent activity. Trends Neurosci 24:455-463. CrossRef Medline

Winawer J, Horiguchi H, Sayres RA, Amano K, Wandell BA (2010) Mapping hV4 and ventral occipital cortex: the venous eclipse. J Vis 10(5):1 1-22. CrossRef Medline

Xing Y, Ledgeway T, McGraw PV, Schluppeck D (2013) Decoding working memory of stimulus contrast in early visual cortex. J Neurosci 33:1030110311. CrossRef Medline 